\title{
Assessment of the Generalizability of an eConsult Service through Implementation in a New Health Region
}

\author{
Clare Liddy, MD, MSc, Isabella Moroz, PhD, Ariana Mihan, and Erin Keely, MD
}

Introduction: Excessive wait times for specialist care are a significant issue in many countries. Electronic consultation (eConsult) services have demonstrated the ability to improve access to specialist care. In this article, we evaluated the implementation of a successful eConsult service in a new jurisdiction to test its generalizability.

Methods: We used a multimethod approach to evaluate the Champlain Building Access to Specialists through eConsultation eConsult service's implementation in the South East Local Health Integration Network of Ontario, Canada. Our quantitative analysis drew on use data collected automatically by the service and survey responses completed between February 1, and June 15, 2017. For our qualitative analysis, we conducted a thematic analysis of 3 focus groups with primary care providers and specialists participating in the pilot study.

Results: Forty-nine out of the potential 219 primary care providers in Kingston submitted 301 cases to 24 specialty groups during the study period. Monthly case volume grew from 15 in February to 90 in May. The most frequently requested specialties included dermatology $(n=59)$, cardiology $(n=27)$, and gastroenterology $(n=26)$. Specialists responded in a median of 2 days, and a referral was originally contemplated but ultimately avoided in $40 \%$ of cases. Providers spoke positively of the service, citing high levels of satisfaction, enhanced collegiality, increased trust, and improved patient flow.

Conclusions: Adoption of the eConsult service in the South East Local Health Integration Network was successful. The service exceeded all adoption targets, and the number of completed cases demonstrated a consistently upward trend, suggesting continued growth beyond the study's duration. The service's rate of adoption, high levels of satisfaction, and use data similar to other regions all demonstrate eConsult's generalizability. (J Am Board Fam Med 2019;32:146-157.)

Keywords: Canada, Electronic Health Records, Personal Satisfaction, Primary Health Care, Referral and Consultation

Barriers in accessing specialist care remain a significant issue in many places. A recent survey by the Commonwealth Fund placed Canada last among 11 countries in terms of access to specialist care. ${ }^{1}$ Although faring better on the Commonwealth Fund survey, the United States and the United Kingdom have their own issues relating to special-

This article was externally peer reviewed.

Submitted 7 June 2018; revised 8 November 2018; accepted 30 November 2018.

From C.T. Lamont Primary Healthcare Research Centre, Bruyère Research Institute, Ottawa, Ontario, Canada (CL, IM, AM); Department of Family Medicine, University of Ottawa, Ottawa, Ontario, Canada (CL); Department of Medicine, University of Ottawa, Ottawa, Ontario, Canada (EK); Division of Endocrinology/Metabolism, The Ottawa Hospital, Ottawa, Ontario, Canada (EK). ist access, including delays and inequities to access. ${ }^{2-4}$ Delays in access have significant consequences for patients, leading to poor health outcomes, decreased ability to carry out day-to-day tasks, and increased mortality. ${ }^{5,6}$

Funding: Funding for this study was provided by OntarioMD, the Ontario Ministry of Health and LongTerm Care, Ontario Telemedicine Network, the South East LHIN, and the Canadian Institutes for Health Research. The funders had no role in study design, data collection/ analysis/interpretation, or preparation of the manuscript.

Conflict of interest: none declared.

Corresponding author: Clare Liddy, MD, MSc, Clinician Investigator, CT Lamont Primary Health Care Research Centre, Bruyère Research Institute, 43 Bruyère St. Annex E, Room 106, Ottawa, ON K1N 5C8 (E-mail: cliddy@bruyere.org). 
In many countries, referrals from primary care providers (PCPs) to specialists are a necessary step for patients to access health resources and represent a critical interface between providers and across settings. Historically, PCPs worked alongside specialists in hospitals and informal consultation was common, often using individual collaborative relationships for patients to access specialist care. ${ }^{7,8}$ Over the years, the referral-consultation process has grown more and more inefficient, with primary and specialty systems operating seemingly in parallel, with little integration. ${ }^{9,10}$ This lack of integration has been shown to contribute to inefficient care and significant dissatisfaction reported by PCPs, specialists, and patients. Despite the wealth of literature on specialty referrals, ${ }^{10-14}$ the criteria and measures by which a referral can be judged as good or bad, relevant or irrelevant, appropriate or inappropriate, have not yet been defined. ${ }^{15,16}$

With the rising costs of health care and dissatisfaction with a fragmented system, many health care innovators have explored ways to ensure equity, improve efficiency, and reduce redundancy. ${ }^{16,17}$ One such effort involves the expansion of electronic communication capabilities, which is creating new venues for the primary care-specialist interface. ${ }^{17,18}$ Many regions have begun implementing electronic consultation (eConsult) services, which use asynchronous communication channels to facilitate prompt, secure communication between PCPs and specialists. Such services have demonstrated the ability to expedite access to specialty advice, lower costs, and increase satisfaction for patients and providers alike. ${ }^{19,20}$ In addition to improving access to care, eConsult services have been praised by PCPs and specialists for their educational value, including their ability to enrich practice-based learning, promote reflection, improve collegiality and professionalism, inform continuing professional development activities, and enhance training programs' teaching of effective communication and care coordination. ${ }^{21,22}$

Our team implemented an eConsult service in the Champlain Local Health Integration Network (LHIN), a health region situated in eastern Ontario, Canada. Initially launched as a small proofof-concept service, it expanded to a full pilot in 2011 and is now supported by provincial and regional funding. Given the service's success in our region, ${ }^{23,24}$ we have engaged in efforts to expand eConsult on a provincial level. To assess eConsult's generalizability, we partnered with Ontario Telemedicine Network (OTN) and OntarioMD to replicate the Building Access to Specialists through eConsultation (BASE) eConsult model in a neighboring LHIN (ie, health region) by using OTN's existing platform.

In this article, we have evaluated the implementation of an eConsult service in the South East LHIN through a combination of use data and focus groups to paint a broad and holistic picture of the service's expansion. Our findings will be of interest to innovators looking to implement or expand their own eConsult services.

\section{Methods \\ Design}

We used a multimethod approach to evaluate the impact of eConsult's implementation in the South East LHIN, including quantitative use data and qualitative focus group data.

\section{Setting}

The South East LHIN had a population of 495,000 people in 2015 and was projected to surpass 500,000 by 2017 . Accounting for 3.6\% of Ontario's population, the South East LHIN is the third least populous LHIN in Ontario as well as the most rural, with $45 \%$ of the population living in rural areas. There were an estimated 526 family doctors in the region as of 2015, excluding those primarily in focused practice or emergency departments, with 219 of these physicians actively practicing in the LHIN's largest city, Kingston, which served as the target setting for adoption of eConsult. ${ }^{25}$

The Southeastern Ontario Academic Medical Organization (SEAMO) was motivated to champion eConsult's implementation in their region and assumed the role of the local host organization for the pilot. SEAMO is an association whose members are the Clinical Teachers' Association of Queen's University, the Kingston Health Sciences Centre, Providence Care, and Queen's University.

\section{The eConsult Service}

The Champlain BASE eConsult service is a secure web-based platform that can be accessed through any device with a web browser. To use the service, PCPs $\log$ in and enter their question into a freetext field, attaching any items they deem might aid the specialist in their response (eg, images and test 
results). PCPs select a specialty group and submit the case, which is allocated to an appropriate specialist by a case assigner. The specialist has 1 week to respond with advice on treatment, a recommendation for a referral, or a request for more information. Conversation can continue back and forth until the PCP closes the case.

\section{Data Sources}

Our study drew on data from 2 sources: use data automatically collected by the service and focus groups conducted with enrolled providers.

\section{Use Data}

Routine use data were collected automatically in real time and included the number of eConsults the PCP has submitted, the specialty group referred to, specialist response time and self-reported time taken to answer the case, and PCP time to close the case. Case outcomes for impact analysis were collected through a brief 5 -field closeout survey completed by PCPs on the conclusion of each case (see Appendix A). The survey asked PCPs to assess (1) the outcome of the eConsult for the patient, (2) whether or not a referral was contemplated before/ after the eConsult, (3) the helpfulness/educational value of the response in guiding PCP's ongoing evaluation or management of the patient, (4) the value of the response in addressing an important clinical problem that should be incorporated into upcoming continuing medical education events, and (5) any additional comments they may have on the service. Descriptive statistics were used to characterize the use data collected.

\section{Focus Groups}

Three focus groups were held to investigate the perspectives and experiences of the PCPs and specialists participating in the pilot study. The groups were held on Tuesday, June 20, 2017 in Kingston, $\mathrm{ON}$, and consisted of 7 to 12 participants each. It was determined that the experience would provide a beneficial opportunity for PCPs and specialists to hear each other's experiences, roles, and perspectives in the eConsult process. Thus, PCPs and specialists were invited to jointly attend a session. Sampling was purposive: participants who had submitted/responded to at least 1 eConsult throughout the pilot were invited to attend through an e-mail sent by the SEAMO team. No relationship existed between participants and focus group leaders be- fore study commencement. Three 2-hour session times were offered to participants, with 2 being in person and as a teleconference session for those who were unable to attend in person. The sessions were moderated by the lead physicians of the Champlain BASE eConsult team: a family physician and a specialist physician. A 14-question script used in previous focus groups was modified and adjusted to provoke discussion among participants (see Appendix B). The sessions were audio recorded, transcribed, and uploaded to NVivo to facilitate qualitative analysis. Verbal consent was obtained by focus group participants in the recording of the sessions.

\section{Targets and Performance Measures}

The targets and performance measures for the project were determined with the project partners and were based on projections from initial work done in Champlain $\mathrm{LHIN}^{23}$ and from a subsequent replication in the Mississauga Halton LHIN. ${ }^{26}$ The collaborating partners agreed on a work plan for implementing an eConsult service on the OTN's existing provincial platform and set the targets to achieve within 6 months of implementation. The scope and targeted numbers was limited based on the funding allocation and spending rules and required reporting dates.

\section{Analyses \\ Quantitative}

Descriptive statistics were used to characterize the use data collected from February 1 to June 15, 2017. Findings were compared with adoption targets for the number of referrers, specialty services offered, and eConsults submitted, which were determined by the leadership teams before launching the pilot.

\section{Qualitative}

Focus group data were thematically analyzed using NVivo software. The data were coded by 1 reviewer, which was then reviewed by and discussed with 2 other team members to further develop themes. A second thorough review was conducted by another team member to ensure rigor. The developed themes were circulated for feedback with the collaborators involved in the pilot. 
Table 1. Comparison of the Adoption Targets Assigned by Leadership Teams for the SEAMO eConsult Service Pilot Period (February 1, 2017 to June 15, 2017) and the Actual Numbers Achieved by the Service during This Period

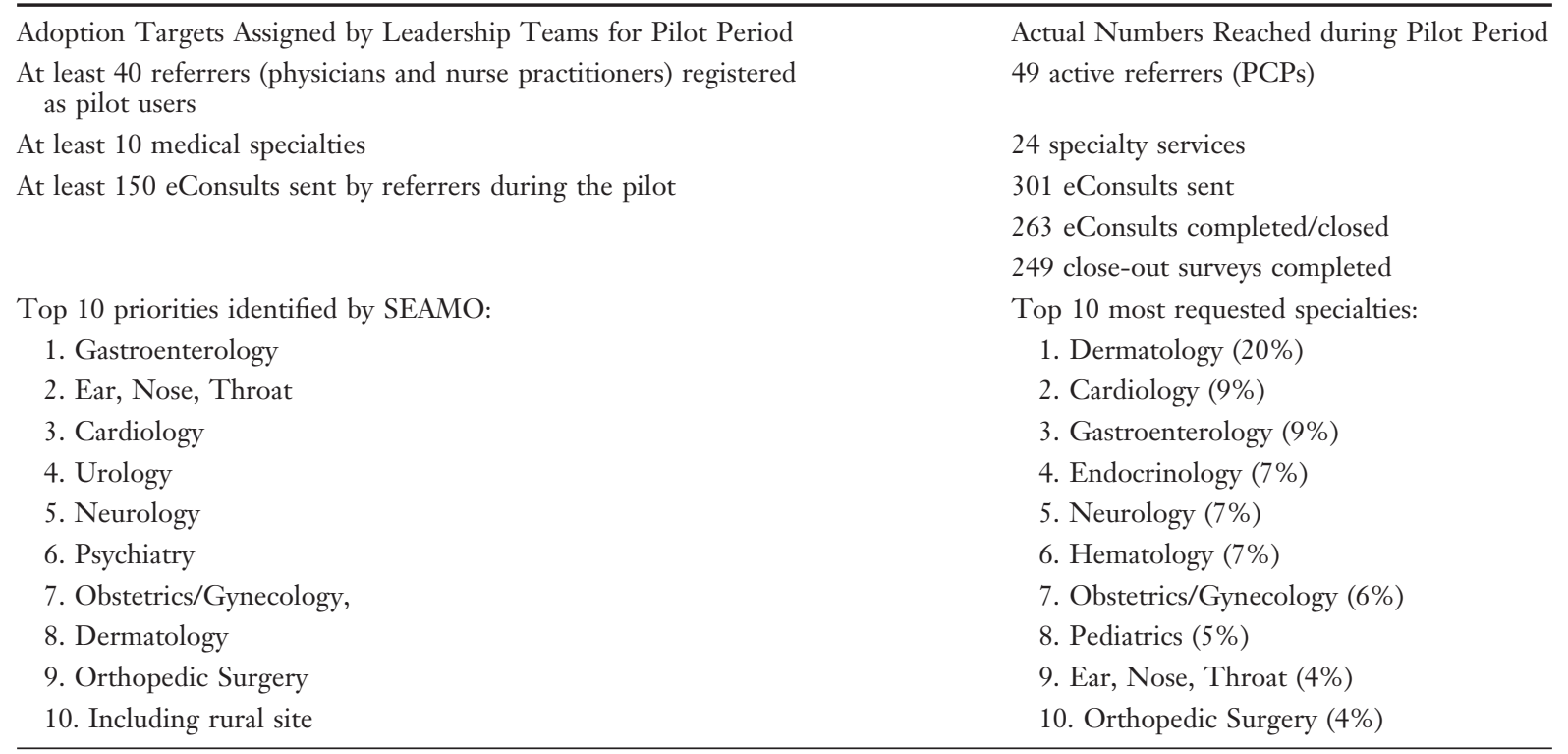

SEAMO, Southeastern Ontario Academic Medical Organization; PCPs, primary care providers.

\section{Ethics}

The Ottawa Health Science Network and Bruyère Research Ethics Boards provided ethics approval for this study.

\section{Results}

Adoption of the eConsult service surpassed adoption targets, and the top specialties requested aligned with the top priorities as identified by SEAMO (Table 1). User adoption exceeded the expected target of 40 referrers, with 49 of the potential 219 PCPs practicing in Kingston submitting at least 1 eConsult (a median number of 5 eConsults submitted per PCP). The majority of participating PCPs came from the targeted Queen's Family Health Team $(n=21)$ and other Family Health Teams and several independent practices in Kingston and the surrounding area as shown in Figure 1.

There were 301 cases submitted to 24 specialty groups from February 1 to June 15, 2017, of which 263 were deemed completed by PCPs as of June $15^{\text {th }}$, and 249 had completed closeout surveys (the remaining cases were completed and surveys sent after the June $15^{\text {th }}$ cutoff). The number of submit-

Figure 1. Distribution of primary care practices that participated in the Southeastern Ontario Academic Medical Organization (SEAMO) pilot of the Champlain Building Access to Specialists through eConsultation (BASE) eConsult service.

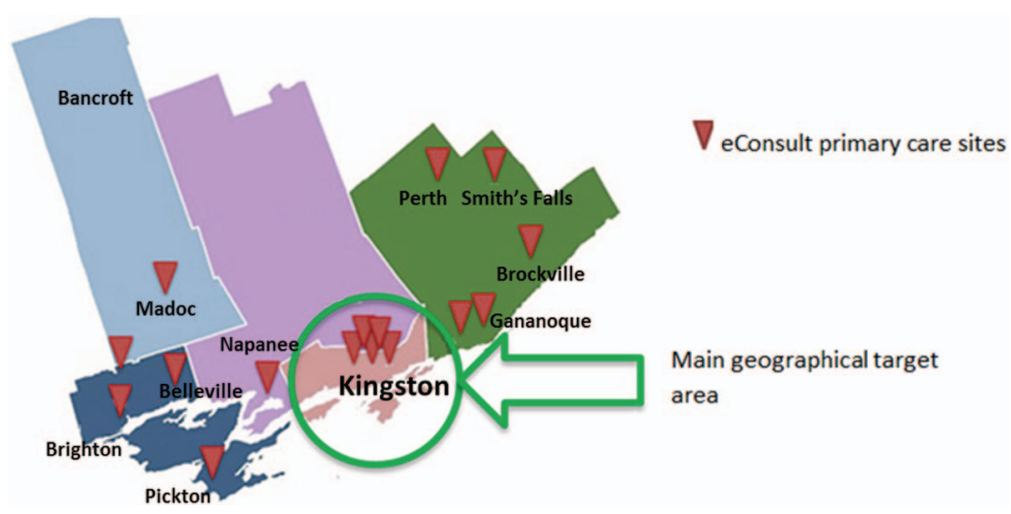


Figure 2. The number of cases submitted through the Champlain Building Access to Specialists through eConsultation (BASE) eConsult service by participants in the Southeastern Ontario Academic Medical Organization (SEAMO) pilot, by month and cumulatively.

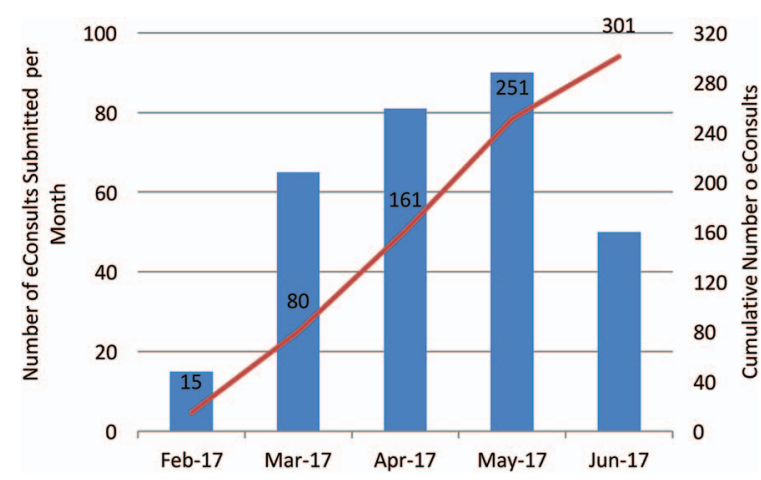

ted cases was more than double the expected target of eConsult cases.

As shown in Figure 2, the volume of eConsults submitted by the PCPs increased each month, from 15 in February to 90 in May (the last month with complete data). The median number of eConsults submitted over the course of the pilot was 5, with an interquartile range of 2 to 9 .

Available specialty groups align well with those identified by SEAMO as their top 10 priorities, with dermatology $(n=59)$, cardiology $(n=27)$, gastroenterology $(n=26)$, endocrinology $(n=21)$, neurology $(n=21)$, and hematology $(n=20)$ being the top 6 most requested specialties.

Specialists provided an initial response to PCP questions in a median of 2 days, with $75 \%$ of eConsults answered within 4 days. In $6 \%$ of cases $(16 / 263)$, the specialists took longer to respond than the prescribed 7-day response period. The self-reported time it took the specialists to complete the eConsult was less than 10 minutes in 32\% of cases, 10 to 20 minutes in $53 \%$ of cases, and more than 20 minutes in $15 \%$ of cases. PCPs closed eConsult cases (eg, received and read response) in a median of 6 days, with $75 \%$ of cases completed within 10 days.

Overall, PCPs received advice on a new or additional course of action in 61\% (153/249) of cases and confirmed their original course of action in $35 \%(86 / 249)$. Only 2\% (4/249) of responses were deemed not to be useful.
In $40 \%$ of cases, a referral was originally contemplated but ultimately avoided. In $18 \%$ of cases a referral was not originally contemplated and was still not needed. Overall, $58 \%$ of all completed cases did not require a face-to-face visit. In $32 \%$ of cases, a referral was originally contemplated and was still needed, but the PCP perceived that eConsult would lead to a more effective specialist visit. In $4 \%$ of cases, a referral was not originally contemplated, but eConsult resulted in a referral being initiated.

The closeout survey question asking PCPs to rate the service's helpfulness and/or educational value used a 5 -point Likert scale ranging from 1 (minimal) to 5 (very valuable). PCPs rated the service at 4 or 5 (indicating valuable and very valuable) in $92 \%$ of cases. Whether the eConsult addresses an important clinical problem that should be incorporated into upcoming continuing medical education events was rated on a scale from 1 (strongly disagree) to 5 (strongly agree). Ratings of 4 or 5 were given in $50 \%$ of cases. Examples of free-text responses from PCPs regarding the benefits of the eConsult system and suggestions for improvement are given in Table 2 .

A total of 11 PCPs and 19 specialists participated in the 3 focus groups, representing a variety of clinics and practices. Participating PCPs represented 4 Family Health Teams and 1 independent practice. Participating specialists represented 11 specialty services: respirology; anesthesiology/chronic pain management; ear, nose, and throat; endocrinology; cardiology; physical medicine and rehabilitation; neurology; urology; dermatology; obstetrics and gynecology; and ophthalmology.

Three themes emerged from the focus group discussions: description of the process, benefits of eConsult, and organization of the service.

\section{Description of the Process}

Participants' discussions of the eConsult process involved a number of factors, including registration and setup, platform, workflow, quality of eConsults, and engaging patients.

\section{Registration and Setup}

The majority of participants found the registration and setup process straightforward. Some participants described joining the service on their own, although most joined during group onboarding sessions. One of the chief benefits of the group on- 
Table 2. Free Text Responses from Closeout Survey Completed by Primary Care Providers using the Southeastern Ontario Academic Medical Organization (SEAMO) eConsult Service

\begin{tabular}{|c|c|}
\hline Specialty Group & Comment \\
\hline \multirow[t]{3}{*}{ Dermatology } & $\begin{array}{l}\text { "Although the referral is still needed, the helpful information from the eConsult allowed me to begin } \\
\text { diagnosis and treatment rather than waiting for him to see the specialist." }\end{array}$ \\
\hline & "Very efficient - thanks for quick response." \\
\hline & "Great system in terms of rapid response to clinical question." \\
\hline \multirow[t]{3}{*}{ Hematology } & "Great system as long as our referral clerk is the steward of it all." \\
\hline & "Simple question for my own learning. eConsult answered my question well, was very educational." \\
\hline & "Would have been a good case for eConsult but another specialist referred to Hematology regardless." \\
\hline Medical Oncology & "I found the process to be very efficient and accessible." \\
\hline \multirow[t]{2}{*}{ Neurology } & "This was my first time using BASE eConsult and I was very satisfied with its utility and ease of use." \\
\hline & $\begin{array}{l}\text { "Patient was presenting with complaint that stumped us, but eConsult mentioned some neuro } \\
\text { differentials I had not thought of. Will be ordering MRI now." }\end{array}$ \\
\hline Neurosurgery & "Very expedient." \\
\hline Obstetrics & "Fast and clinically useful feedback." \\
\hline Orthopedic Surgery & "Great service, answer was concise and short, good explanation. There was no need to see the patient." \\
\hline \multirow[t]{2}{*}{ Pediatrics } & "Really great advice on an unusual presentation." \\
\hline & $\begin{array}{l}\text { "What an incredible service. So helpful to be able to ask a quick question and get a specialist's } \\
\text { opinion. Please keep this service going." }\end{array}$ \\
\hline \multicolumn{2}{|c|}{ Suggestions for Improvement } \\
\hline \multicolumn{2}{|c|}{$\begin{array}{l}\text { "One of my three security questions is asking for a month and a date of a birthday. I know the month and date but can't seem } \\
\text { to figure out which format the system wants ... I've tried multiple different combinations of year and month ... may be } \\
\text { specifying how the system wants the month and date input??" }\end{array}$} \\
\hline \multicolumn{2}{|c|}{$\begin{array}{l}\text { "The question was a straightforward one regarding medication options for a particular condition. The specialist declined to } \\
\text { comment as the patient was scheduled to see another specialist (different specialty) in the future." }\end{array}$} \\
\hline \multicolumn{2}{|c|}{$\begin{array}{l}\text { "Main challenge I have with this service is that I forget about it and I have to log in to a new site each time to use it.... when } \\
\text { will it be incorporated to all EMRs?" }\end{array}$} \\
\hline \multicolumn{2}{|c|}{$\begin{array}{l}\text { "It would be helpful if I could see a list of local specialists who provide eConsult and direct my consult directly to one of } \\
\text { them. . when I tried to scroll the list, Dr X was not even listed." }\end{array}$} \\
\hline
\end{tabular}

BASE, Building Access to Specialists through eConsultation; MRI, magnetic resonance imaging; EMR, electronic medical record.

boarding session, according to participants, was having an expert there to walk through the process in person: "I thought it was really helpful that someone came [to the clinic] at a time that was prearranged and walk us through the whole process." A few participants encountered technical issues that caused short-term frustration but were ultimately resolved.

\section{Platform}

Participants discussed the different ways in which they accessed the eConsult platform. Although some participants accessed it directly through a web browser, many used the version integrated with their electronic medical records (EMRs). These participants described some issues regarding formatting or compatibility with their software or hardware, as well as glitches such as pages timing out before completion. However, their overall impression with the platform was favorable, with several participants noting that it was user friendly and required minimal training: "The whole system was just so intrinsic to use. You do not really need extensive training. You could just figure it out very quickly."

\section{Workflow}

Participants described a variety of workflows. Some participants set aside blocks of time after their office hours, whereas others completed them at the time of the patient's appointment. This variation was described by PCPs and specialists alike, with no one method predominating in either group. In several cases, PCPs submitted their cases via delegates, who entered their questions into the online application and submitted them on their behalf. In these cases, participants noted that they "essentially just do it [complete an eConsult] as a normal consult," which resulted in minimal disruption to their established workflow. As 1 PCP described: "We've just set up a very quick template within our EMR. So it is really point of care, which is nice. The 
patient is there as I am typing the question and I read it with them, and they have a sense of what we're asking."

\section{Quality of eConsults}

Participants consistently described the questions and advice they received through eConsult as being of extremely high quality. They noted that the advice they received had a tangible benefit to the care they provided patients, and agreed that it had allowed many patients to avoid an unnecessary specialist visit: "I can say that the quality of consults that we received has been stunning. They have been fantastic. In a number of cases actually it is facilitated triage because if somebody is referred for vague symptoms, they tend to get moved toward a general stream." Participants stated that eConsult was especially valuable for complex cases, where the patient's history or multimorbidities make treating the patient in primary care difficult:

When you think of 1 condition, you know, 'this patient has this thing.' But the patients we have and that you refer about, have 55 things wrong with them, all competing. It is a very specific thing because of all the complexity of these multiple overlapping issues, whether it be treatments or diseases.

\section{Engaging Patients}

Several participants described how they engaged patients in the eConsult process. In most cases, participants discussed the eConsult with patients before sending it, although some described cases where the patient was unaware of the eConsult until they received the results. On receiving an answer to the eConsult, most participants contacted patients by telephone or communicated the results at a follow-up visit.

\section{Benefits of eConsult}

Participants cited a number of benefits of using eConsult, including improved access to specialist advice, avoiding unnecessary referrals, education, expanded scope of practice, improved care for rural and underserved populations, and increased patient and provider satisfaction.

\section{Improved Access to Specialist Advice}

When discussing the benefits of eConsult, participants frequently noted its ability to improve patients' access to specialist care. Participants men- tioned the brief turnaround times between posing a question and receiving a response, with 1 participant stating: "It is quicker than having to wait on hold for a specialist sometimes." Participants also describe the service's efficiency, noting its ability to reduce the burden of multiple appointments and delays that some patients face when attempting to access specialist care: "it was so efficient, for patients to get an answer and for our family physicians to give an answer, help them out, get the patient done, keep observing and save a lot of travel back and forth."

\section{Avoiding Unnecessary Referrals}

Several participants stated that eConsult had allowed patients to avoid unnecessary specialist referrals. One participant described a case where a patient had wanted a second opinion regarding a prescribed ankle surgery, which the participant was able to secure via eConsult. Had he referred using the traditional method, the patient would have likely waited months for a response, delaying his ability to seek treatment. Even in cases where a referral was ultimately needed, participants agreed that eConsult still had value, as it allowed providers to ensure the patient saw the right specialist and had the necessary work-up before the appointment.

\section{Education}

A recurring theme among participants' discussions of eConsult was its educational value. Several participants who worked in primary care practices stated that sending eConsults and reading specialists' replies had provided them with knowledge they could apply to other patients in the future. These participants also praised the extra effort specialists made to provide educational materials, such as studies or guidelines, and noted that eConsults "often spark discussion points with the residents as well and can actually be a segue into a really nice educational opportunity for all us." Several participants mentioned that eConsult cases could be used to inform continuing medical education curricula.

\section{Expanded Scope of Practice}

A few participants argued that another advantage of eConsult was its ability to help PCPs retain and treat patients with more complex cases. Participants described the ability to consult with allied 
health professionals as one facet of this. One participant expounded on the benefits of a shared care approach:

My impression from previous conversations with family physicians is that they really want to look after those patients because they have a relationship with them and they want to maintain it. [...] The concept of shared care, where we use the eConsult to provide the information that you need and let you carry on and knowing that if it does not work out you can just send them back or whatever, to me that makes a lot of sense.

\section{Improved Care for Rural and Underserved Populations}

When discussing eConsult's ability to improve access and avoid unnecessary referrals, participants emphasized that these benefits were particularly valuable for patients from remote or underserved populations. Several participants raised the example of rural patients, many of whom face financial or geographic limitations in their ability to travel to urban centers where specialists often practice. One participant described seeing patients who had traveled from remote northern communities by plane for care that could easily have been offered through eConsult: "it is crazy the cost of sending these people down here. I just go what, are you kidding? [...] They are going to fly them all the way down from James Bay and then they are just treating them with diet?" Participants also recommended focusing on "not just patient location but patient characteristic," citing patients with low socioeconomic status, cognitive issues, or those incarcerated in penitentiaries as groups who could benefit from eConsult.

\section{Increased Patient and Provider Satisfaction}

Participants described the high level of satisfaction eConsult had from patients and providers alike. Many participants stated that the patients they had treated using eConsult "have been very enthusiastic and receptive," and were impressed with how quickly they received answers to the questions sent on their behalf. Likewise, participants themselves regarded the service highly, with several noting that it was held in high esteem by them and their colleagues: "We just think it is the best thing ever, and if there's any chance of keeping this going, I am good."

\section{Organization of the Service}

Lastly, participants discussed their attitudes and preferences toward how the service is organized and offered suggestions as to how it should adapt when expanding the service province-wide. Subthemes in this area were governance, remuneration, specialties available, volume of cases, and community of practice.

\section{Governance}

In terms of governance, participants stated that it was important to ensure a wide range of voices, particularly patient voices, were heard and could contribute to the service's oversight. One participant suggested establishing "a small skills-based board, 7 to eleven people. So some users both on the primary care side and the specialist side. So technical people who know how the back end works. And then some strategic people who are politically connected. And then 1 or 2 patients." Overall, however, participants agreed that the structure of the service as it currently stood was working and did not require extensive revision when expanding provincially: "We probably do not need to reinvent the wheel here, [...] that latching onto the existing admin structure would probably work pretty well."

\section{Community of Practice}

Although broadly supportive of eConsult's expansion, participants frequently emphasized the value of retaining a community focus within a broader framework. Participants stressed that they appreciated how eConsult connected them with other providers in their region, giving them "a sense of playing on the same team." They feared that a provincial service could dilute the communities of practice that had emerged through eConsult, reducing the benefit of collegiality it fostered:

There's a big difference between an eConsult that comes from someone you know to someone you know, versus sending it into the ether. When I've been doing these and everyone's name pops up, it is, like, "hey, I know that person." [...] but if it is coming from North Bay, I may not feel that same enthusiasm.

\section{Remuneration}

When discussing remuneration for eConsult, many of the participants were unclear on how it worked or how much they would be paid, as they had only 
recently joined the pilot. Of those who were aware of the payment structure (and those who were not and were informed at the focus group), the majority felt that the current rate and method of compensation was fair. One participant noted that "[among] several of the physicians I've spoken to who do this, the remuneration was not a consideration for doing it," although he clarified that it might become a bigger issue if the volume of cases increased. Another PCP argued that compensation for eConsults was appreciated but unnecessary, as "it falls within what I would expect to need to do for my patients anyway."

\section{Specialties Available}

A few participants made suggestions about the types of specialty groups available through eConsult, and ways to expand the service's utility. One participant suggested adding midwives to the service, while another raised the possibility of specialist to specialist referrals. Participants noted that allowing multidisciplinary teams to collaborate on a patient's case would benefit patients with complex conditions requiring care from multiple providers.

\section{Volume of Cases}

When discussing eConsult's expansion, a common theme was the potential effect that a higher volume of cases might have on how the service functions. Participants noted that at their current frequency, eConsults could be easily managed on top of their regular clinic work, but "Ultimately there would be a problem if it gets too many and if it is not split evenly among your colleagues. If there's only 1 person doing them or 2, then it might become [an issue]."

\section{Discussion}

Clinics in the South East LHIN successfully adopted eConsult, with all target numbers for user adoption being surpassed within 4.5 months of the pilot being launched. Providers spoke positively of the service, citing high levels of patient satisfaction. The recruitment of PCPs and specialists together, maintaining the community of practice, was highly appreciated, with participants describing advantages, such as enhanced collegiality, increased trust, and facilitated patient flow. Specialists responded to PCPs in a median of 2 days, and $40 \%$ of cases resulted in PCPs avoiding a referral they had orig- inally considered. These findings closely resemble those of studies conducted in other regions where eConsult has been implemented. For example, our earlier studies of PCPs and specialists participating in eConsult service in Champlain LHIN revealed that all providers recognized and appreciated the educational value of eConsult and often cited it as a motivator for continuing to participate..$^{22,27}$ The direct communication between PCPs and specialists enabled by eConsult is often missing in the traditional face-to-face consultations and has been found to improve dialog between PCPs and specialists and maximize the efficiency, safety, and quality of specialty health care delivery within a patient-centered medical home-neighborhood. ${ }^{18} \mathrm{~A}$ study of eConsult's implementation in the Mississauga Halton LHIN found a median response time of 1.1 days, whereas an examination of all cases completed over the first 5 years of the service (over 14,000 in total) revealed a median response time of 0.9 days. The rate of unnecessary referral avoidance remained unchanged at $40 \%$ across all 3 studies. The consistency of these findings showcases the service's generalizability across multiple settings.

The challenges associated with scaling up health care innovations are well-reported..$^{28}$ Researchers have pinpointed several barriers to scale-up, including providers' reluctance to change behaviors in care delivery, ${ }^{29}$ difficulties spreading knowledge within organizations, ${ }^{30,31}$ and limitations of infrastructure. ${ }^{32}$ This is especially true in Canada, which has been described as a "land of perpetual pilot projects," in which promising innovations are implemented at considerable cost only to languish at a regional level, unable to expand beyond the pilot phase. ${ }^{33,34}$ This trend is costly, as innovations must continually shoulder the up-front expenses of implementation and results in new knowledge remaining siloed and unavailable to a wider audience that could benefit from it. ${ }^{34}$ Given these issues, our findings are encouraging, as they suggest a high level of generalizability for eConsult and can serve as a valuable blueprint for expansion to new jurisdictions. However, it is worth noting several limitations when attempting this interpretation. Implementation at both sites occurred within the same province, which makes it unable to account for the challenges associated with interprovincial expansion. ${ }^{33}$ Furthermore, implementation relied on an academic lead site, which may be more responsive to innovations and will not be available in all con- 
texts. Methodological limitations related to study duration and selection of focus group participants with inherent selection and desirability biases must be also acknowledged. Our team is aware of the challenges associated with eConsult's expansion and has previously examined the Canadian policy landscape to identify areas where legislation could impede eConsult's growth, revealing 3 aspects of concern: payment, privacy, and interjurisdictional licensing. ${ }^{35}$ In an effort to overcome these obstacles, we applied an integrated knowledge translation strategy that culminated in a National eConsult Policy Think Tank, which convened experts and stakeholders from 9 Canadian provinces/territories. ${ }^{36}$ It is our hope that these efforts will support the further expansion of the service to new jurisdictions across Canada.

\section{Conclusion}

Adoption of the eConsult service in the South East LHIN was successful. The service exceeded all adoption targets, and the number of completed cases demonstrated a consistently upward trend, suggesting continued growth beyond the study's duration. The service's rate of adoption, high levels of provider satisfaction, and usage data similar to other regions all demonstrate eConsult's generalizability.

The authors wish to thank the PCPs and specialists who participated in the service; Lacey Cranston, Danielle Claus, and Gina Johar for their assistance in expanding eConsult to the South East LHIN and conducting the study; and Justin Joschko for his help with drafting the manuscript and preparing it for publication.

To see this article online, please go to: http://jabfm.org/content/ 32/2/146.full.

\section{References}

1. Canadian Institute for Health Information. How Canada compares: results from The Commonwealth Fund's 2016 international health policy survey of adults in 11 countries. Available from: https:/www.cihi.ca/ sites/default/files/document/text-alternative-version2016-cmwf-en-web.pdf. Published 2017. Accessed May 4, 2018.

2. Schneider EC, Sarnak DO, Squires D, Shah A, Doty MM. Mirror, mirror 2017: international comparison reflects flaws and opportunities for better U.S. health care. New York: The Commonwealth Fund; 2017.

3. Cook NL, Hicks LS, O'Malley AJ, Keegan T, Guadagnoli E, Landon BE. Access to specialty care and medical services in community health centers. Health Aff 2007;26:1459-68.

4. Decker SL. In 2011 nearly one-third of physicians said they would not accept new Medicaid patients, but rising fees may help. Health Aff (Millwood) 2012;31:1673-9.

5. Canadian Institute for Health Information. Health Care in Canada, 2012: a focus on wait times. Ottawa: Canadian Institute for Health Information; 2012.

6. Day B. The consequences of waiting. In: Globerman $\mathrm{S}$, editor. Reducing wait times for health care: what Canada can learn from theory and international experience. Vancouver, BC: Fraser Institute; 2013. P. 45-76.

7. Bichel A, Erfle S, Wiebe V, Axelrod D, Conly J. Improving patient access to medical services: preventing the patient from being lost in translation. Healthc Q 2009;13:61-8.

8. Long D, Iedema R, Lee BB. Corridor conversations: clinical communication in casual spaces. In: Iedema $\mathrm{R}$, editor. The discourse of hospital communication. Springer; 2007. P. 182-200.

9. Mehrotra A, Forrest CB, Lin CY. Dropping the baton: specialty referrals in the United States. Milbank Q 2011;89:39-68.

10. O'Malley AS, Reschovsky JD. Referral and consultation communication between primary care and specialist physicians: finding common ground. Arch Int Med 2011;171:56-65.

11. O'Donnell CA. Variation in GP referral rates: what can we learn from the literature? Fam Pract 2000; 17:462-71.

12. Chan BT, Austin PC. Patient, physician, and community factors affecting referrals to specialists in Ontario, Canada: a population-based, multi-level modelling approach. Med Care 2003;41:500-11.

13. Forrest CB, Majeed A, Weiner JP, Carroll K, Bindman AB. Comparison of specialty referral rates in the United Kingdom and the United States: retrospective cohort analysis. BMJ 2002;325:370-1.

14. Forrest CB, Nutting PA, von SS, Rohde C, Starfield B. Primary care physician specialty referral decision making: patient, physician, and health care system determinants. Med Decis Making 2006;26:76-85.

15. Davies P, Pool R, Smelt G. What do we actually know about the referral process? Br J Gen Pract 2011;61:752-3.

16. Guevara JP, Hsu D, Forrest CB. Performance measures of the specialty referral process: a systematic review of the literature. BMC Health Serv Res 2011; $11: 1$.

17. Kirsh SR, Ho PM, Aron DC. Providing specialty consultant expertise to primary care: an expanding spectrum of modalities. Mayo Clin Proc 2014;89: 1416-1426.

18. Tuot DS, Murphy EJ, McCulloch CE, Leeds K, Chan E, Chen AH. Leveraging an electronic referral 
system to build a medical neighborhood. Healthc (Amst) 2015;3:202-8.

19. Liddy C, Drosinis P, Keely E. Electronic consultation systems: worldwide prevalence and their impact on patient care-a systematic review. Fam Pract 2016;33:274-85.

20. Vimalananda VG, Gupte G, Seraj SM, Orlander J, Berlowitz D, Fincke BG, et al. Electronic consultations (e-consults) to improve access to specialty care: a systematic review and narrative synthesis. J Telemed Telecare 2015;21:323-30.

21. Keely E, Archibald D, Tuot D, Lchonan H, Liddy C. Unique educational opportunities arising from electronic consultation services. Acad Med 2017;92: 45-51.

22. Liddy C, Afkham A, Drosinis P, Joschko J, Keely E. Impact and satisfaction with a new eConsult service: a mixed methods study of primary care providers. J Am Board Fam Med 2015;28:394-403.

23. Keely E, Liddy C, Afkham A. Utilization, benefits, and impact of an e-consultation service across diverse specialties and primary care providers. J Telemed E Health 2013;19:733-8.

24. Joschko J, Liddy C, Moroz I, et al. Just a click away: exploring patients' perspectives on receiving care through the Champlain BASE eConsult service. Fam Pract 2018;35:93-8.

25. Ontario Physician Human Resources Data Centre. Physicians in Ontario 2015: Report from the Active Physician Registry. Available from: http://www.ophrdc. org/wp-content/uploads/2016/08/2015-AnnualReport-Physicians-in-ONTARIO-PIO.pdf. Published August 2015. Accessed October 4, 2018.

26. Liddy C, Moroz I, Afkham A, Keely E. Evaluating the implementation of the Champlain BASE
eConsult Service in a new region of Ontario, Canada: a cross-sectional study. Healthc Pol 2017;13: 79-95.

27. Keely E, Drosinis P, Afkham A, Liddy C. Perspectives of Champlain BASE specialist physicians: their motivation, experiences and recommendations for providing eConsultations to primary care providers. Stud Health Technol Inform 2015;209:38-45.

28. Berwick DM. The science of improvement. JAMA 2008;299:1182-4.

29. Grol R, Grimshaw J. From best evidence to best practice: effective implementation of change in patients' care. Lancet 2003;362:1225-30.

30. Rogers E. Diffusion of Innovations. New York: Free Press; 2003.

31. Berwick DM. Disseminating innovations in health care. JAMA 2003;289:1969-75.

32. Berwick DM. Lessons from developing nations on improving health care. BMJ 328:1124, 2004.

33. Bégin HM, Eggertson L, Macdonald N. A country of perpetual pilot projects. Can Med Assoc J 180: 1185, 2009.

34. Naylor D, Girard F, Mintz JM, et al. Unleashing innovation: excellent healthcare for Canada: report of the advisory panel on healthcare innovation. Ottawa, ON: Government of Canada; 2015.

35. Liddy C, Joschko J, Keely E. Policy innovation is needed to match health care delivery reform: the story of the Champlain BASE eConsult service. Health Reform Observer 2015;3:1-11.

36. Liddy C, Moroz I, Joschko J, et al. Using an integrated knowledge translation (IKT) approach to enable policy change for electronic consultations in Canada. Healthc Policy 2018;14:19-29. 


\section{Appendix A}

Mandatory survey completed by primary care providers at the conclusion of each eConsult case, October 2016 to Present

\section{Q1: Which of the following best describes the} outcome of this eConsultation for your patient?

1) I was able to confirm a course of action that I originally had in mind

2) I got new advice for a new or additional course of action

3) I did not find the response very useful

4) None of the above

\section{Q2: As a result of the eConsultation would you say}

that:

1) Referral was originally contemplated but now avoided at this stage

2) Referral was originally contemplated and is still needed-this eConsult likely leads to a more effective visit
3) Referral was not originally contemplated and is still not needed-this eConsult provided useful feedback/instruction

4) Referral was not originally contemplated, but eConsult process resulted in a referral being initiated

5) Other (please explain)

\section{Q3: How belpful and/or educational was this} response in guiding your ongoing evaluation or management of the patient?

Minimal 12345 Excellent

Q4: This eConsult addresses an important clinical problem that should be incorporated into upcoming continuing medical education events:

1) Strongly Disagree

2) Disagree

3) Neutral

4) Agree

5) Strongly Agree

Q5: We would value any additional feedback you provide: 